\title{
Exopolysaccharides and Antimicrobial Biosurfactants Produced by Paenibacillus macerans TKU029
}

\author{
Tzu-Wen Liang • Chia-Chen Wu • Wei-Ting Cheng • \\ Yu-Chi Chen • Chuan-Lu Wang • I-Li Wang • \\ San-Lang Wang
}

Received: 16 July 2013 / Accepted: 30 September 2013 /

Published online: 15 October 2013

(C) Springer Science+Business Media New York 2013

\begin{abstract}
Paenibacillus macerans TKU029 can produce exopolysaccharides (EPSs; $3.46 \mathrm{~g} / \mathrm{L})$ and a biosurfactant $(1.78 \mathrm{~g} / \mathrm{L})$ in a medium with $2 \%(w / v)$ squid pen powder as the sole carbon/nitrogen source. The biosurfactant can reduce the surface tension of water from 72.30 to $35.34 \mathrm{mN} / \mathrm{m}$ at a concentration of $2.76 \mathrm{~g} / \mathrm{L}$ and reach an emulsification index of $56 \%$ after a 24 -h reaction with machine oil. This biosurfactant is stable at $121{ }^{\circ} \mathrm{C}$ for $20 \mathrm{~min}$, over a $\mathrm{pH}$ range from 3 to 11 , and in $<5 \%$ salt solutions. It also shows significant antimicrobial activity, which remains active after treatment at $121{ }^{\circ} \mathrm{C}$ and at $\mathrm{pH}$ values from 4 to 10, against Escherichia coli BCRC13086, Staphylococcus aureus BCRC10780, Fusarium oxysporum BCRC32121 and Aspergillus fumigatus BCRC30099. Furthermore, human skin shows from 37.3 to $44.3 \%$ hydration after being treated with TKU029 EPSs for $180 \mathrm{~min}$. These results imply that EPSs and the biosurfactant from this strain have potential in cosmetics, for removal of oil contamination, and as antimicrobial agents.
\end{abstract}

Keywords Paenibacillus macerans $\cdot$ Exopolysaccharides · Biosurfactant $\cdot$ Antimicrobial activity $\cdot$ Skin hydration $\cdot$ Squid pen

T.-W. Liang $\cdot$ S.-L. Wang

Life Science Development Center, Tamkang University, New Taipei City 25137, Taiwan

T.-W. Liang $\cdot$ C.-C. Wu $\cdot$ W.-T. Cheng $\cdot$ S.-L. Wang $(\bowtie)$

Department of Chemistry, Tamkang University, New Taipei City 25137, Taiwan

e-mail: sabulo@mail.tku.edu.tw

Y.-C. Chen

Department of Cosmetic Science, Vanung University, Chung-Li 320, Taiwan

C.-L. Wang

Department of Cosmetic Science and Application, Lan-Yang Institute of Technology, I-Lan 261, Taiwan

I.-L. Wang

Department of Chemical and Materials Engineering, Tamkang University, New Taipei City 25137, Taiwan

Present Address:

S.-L. Wang

No. 151, Yingchuan Rd., Tamsui Dist., New Taipei City 25137, Taiwan 


\section{Introduction}

Biosurfactants are a class of surfactants mainly produced by microorganisms [1]. Most biosurfactant-producing bacteria are isolated from oil-containing media such as waste oil, oil-contaminated soil and sludge [2-9]. Biosurfactants can increase the availability of insoluble carbon sources. Organisms produce biosurfactants to dissolve and utilise insoluble carbon for survival [10]. The action of surfactants is due to a combination of active surface molecules with different chemical moieties; therefore, most surfactants are produced by chemical synthesis. In recent years, interest in the advantages of biosurfactants has increased. These advantages include low toxicity, high biodegradability, good environmental compatibility, high foaming capacity, high selectivity and stability in extreme environments [11]. Over the last decade, biosurfactants have been under investigation as potential replacements for synthetic surfactants and are expected to have many potential industrial and environmental applications related to emulsification, foaming, detergency, wetting, dispersion and solubilisation of hydrophobic compounds [12]. Additionally, over the past few decades, the number of known exopolysaccharides (EPSs) produced by microbial fermentation has been gradually increasing. In recent years, microbial EPSs have found many applications in the food, pharmaceutical and other industries as they have different physiological activities from natural gums and synthetic polymers [13,14]. Moreover, they are highly susceptible to natural biodegradation and are less harmful than synthetic polymers.

Microbial communities of Acinetobacter, Arthrobacter, Pseudomonas, Halomonas, Bacillus, Rhodococcus, Enterobacter and yeast have been reported to produce biosurfactants and EPSs [12]. Most biosurfactants are produced by the metabolic activities of yeast or bacteria on substrates such as sugars, lipids, alkanes and waste material [15]. Molecular structures of biosurfactants include chemical groups such as glycolipids, lipopeptides, phospholipids, neutral lipids and other materials [16-18]. The lipopeptides are an interesting class of microbial surfactants because of their manifold attractive properties, such as antibiotic activity [19]. Few reports are available about the simultaneous production of biosurfactants and EPSs from the same microorganisms [12]. The present study reports the production of an antimicrobial biosurfactant and moisturising EPSs by Paenibacillus macerans TKU029 with squid pen powder (SPP) as the sole C/N source. The annual catch of the swordtip squid is approximately $10,000 \mathrm{mt}$, according to Japanese and Chinese fishing data [20]. Of that, approximately $100 \mathrm{mt}$ (or $1 \%$ ) was squid pen. The price of SPP is very low (about US $\$ 0.30$ per kilogram). The production of inexpensive biosurfactant and EPSs is an important factor in the utilisation of fishery processing wastes. The discovery of inexpensive biosurfactant and EPSs not only solves environmental problems but also promotes the economic value of marine wastes. In this study, media and culture conditions for production are optimised, and the characterisation of the biosurfactant and EPSs is discussed. We also report the stability and the potential applications of the biosurfactant and EPSs for removal of oil, antimicrobial activity and moisture retention by human skin.

\section{Methods and Materials}

Materials

SPP and shrimp head powder (SHP) were prepared as described previously [21]. Squid pen and shrimp head were purchased from the Shin-Ma Frozen Food Co. (I-Lan, Taiwan), washed thoroughly with tap water and then dried. The dried materials were ground to a 
powder for use as the carbon/nitrogen source in the production of biosurfactants. All other reagents used were of the highest grade available.

\section{Identification of Microorganism}

The bacterial strain TKU029 was identified on the basis of morphological, physiological and biochemical parameters and on the basis of $16 \mathrm{~S}$ rDNA-based sequence analysis after PCR amplification and cloning. The DNA sequences obtained were compiled and compared with sequences in the GenBank database using the BLAST program. Further identification of strain TKU029 was performed using the analytical profile index (API).

Strain TKU029 was grown on nutrient agar plates. Bacterial cells on the agar surface were resuspended by gentle mechanical agitation in $2 \mathrm{~mL}$ of sterile distilled water. This bacterial suspension was used to inoculate API 50 CHB strips (identification of Bacillus; ATB System, bioMérieux SA, Marcy-I'Etoile, France) following the manufacturer's instructions. The strips were incubated at $30{ }^{\circ} \mathrm{C}$ and observed after 16, 24, 40 and $48 \mathrm{~h}$ to check them against the API identification index and database.

Culture Conditions for Biosurfactant and EPS Production

\section{Concentration of Carbon/Nitrogen Source}

SPP $(0.5-2 \mathrm{~g})$ was added to $100 \mathrm{~mL}$ of basal medium (containing $0.1 \% \mathrm{~K}_{2} \mathrm{HPO}_{4}$ and $0.05 \%$ $\left.\mathrm{MgSO}_{4} \cdot 7 \mathrm{H}_{2} \mathrm{O}\right)$. P. macerans TKU029 was inoculated and the bacteria were cultured at $37^{\circ} \mathrm{C}$ and $150 \mathrm{rpm}$ for 7 days. The centrifuged fermentation broth was measured for surface tension and total sugar content. After analysis, the carbon/nitrogen source concentration that enabled maximal production of biosurfactants and EPSs was selected. The effect of different culture volumes on the production of biosurfactants and EPSs was then investigated.

\section{Culture Volume}

The degree of aeration in the culture medium affects cell growth, and the culture volume is related to the aeration of the medium. Thus, medium containing $2 \%(w / v)$ SPP, $0.1 \%$ $\mathrm{K}_{2} \mathrm{HPO}_{4}$ and $0.05 \% \mathrm{MgSO}_{4} \cdot 7 \mathrm{H}_{2} \mathrm{O}$ was used to investigate the influence of medium volume on the production of biosurfactants and EPSs. Different volumes (50, 100 and $200 \mathrm{~mL}$ ) of medium were poured into individual 250 -mL flasks. Bacterial liquid cultures were grown at $37{ }^{\circ} \mathrm{C}$ and $150 \mathrm{rpm}$ for 6 days to study the effect of medium volume on TKU029 biosurfactant and EPS production. The culture volumes that were most favourable for the production of biosurfactants and EPSs were selected.

\section{Culture Temperature and Medium $\mathrm{pH}$}

Conical flasks containing $100 \mathrm{~mL}$ of medium (consisting of $2 \%(w / v) \mathrm{SPP}, 0.1 \% \mathrm{~K}_{2} \mathrm{HPO}_{4}$ and $0.05 \% \mathrm{MgSO}_{4} \cdot 7 \mathrm{H}_{2} \mathrm{O}$ ) were used to investigate the influence of various temperatures $\left(25,30\right.$, or $\left.37^{\circ} \mathrm{C}\right)$ on TKU029 biosurfactant and EPS production. The temperatures most favourable for biosurfactant and EPS production were selected. To determine the optimum initial medium $\mathrm{pH}$ for TKU029 biosurfactant and EPS production, the medium was adjusted to the appropriate $\mathrm{pH}$ by the addition of $1 \mathrm{~N} \mathrm{HCl}$ or $1 \mathrm{~N} \mathrm{NaOH}$ prior to sterilisation. A time course experiment was performed in a $250-\mathrm{mL}$ flask containing the optimised culture medium based on the results of the single-factor experiments. 
Measurement of Surface Tension

Surface tension was measured at room temperature using a contact angle goniometer (Sindatek Model 100SB) with the pendant drop method. Model 100SB is an instrument with a compact design for the contact angle measuring technique and drop shape analysis. For software-controlled measurement and analysis of the drop shape according to the pendant drop method, the surface tension is determined by analysis of the drop shape.

\section{Measurement of Total Sugar}

To evaluate total sugar in the medium, the phenol-sulphuric acid method was used [22]. Briefly, $25 \mu \mathrm{L}$ of $5 \%$ phenol was added to $1 \mathrm{~mL}$ of sample. After shaking, $2.5 \mathrm{~mL}$ of concentrated $\mathrm{H}_{2} \mathrm{SO}_{4}$ was added. The mixture was left to stand for $10 \mathrm{~min}$ and absorbance was read at $490 \mathrm{~nm}$. Pure D-glucose was employed as a standard.

\section{Isolation of TKU029 Biosurfactant}

The culture broth was centrifuged at $10,400 \times g$ for 15 min to obtain a cell-free supernatant. The TKU029 biosurfactant was precipitated by adjusting the $\mathrm{pH}$ of the cell-free supernatant to 12 using $2 \mathrm{~N} \mathrm{NaOH}$ and by incubating overnight at $4{ }^{\circ} \mathrm{C}$. The precipitate thus formed was removed by centrifugation $\left(10,400 \times g, 15 \mathrm{~min}, 4^{\circ} \mathrm{C}\right)$. The supernatant was lyophilised and extracted with methanol. The insoluble substances were removed, concentrated by vacuum drying and used for further analysis.

\section{Isolation of EPSs}

After fermentation, the sample was immediately autoclaved for $20 \mathrm{~min}$ to reduce the ropy condition of the culture and centrifuged $(12,000 \times \mathrm{g}$ for $20 \mathrm{~min})$ to remove the remaining SPP and biomass. The supernatant was filtered through a $0.45-\mu \mathrm{m}$ membrane filter, mixed with two volumes of methanol, stirred vigorously and kept overnight at $4{ }^{\circ} \mathrm{C}$. The precipitate from the methanol dispersion was collected by centrifugation at $12,000 \times \mathrm{g}$ for $15 \mathrm{~min}$, re-dissolved in distilled water and lyophilised to yield the crude EPSs.

\section{Deproteinisation of EPSs}

The crude EPSs were re-dissolved in distilled water and stirred vigorously at $80{ }^{\circ} \mathrm{C}$ for $30 \mathrm{~min}$, mixed with four volumes of anhydrous ethanol, stirred vigorously and kept overnight at $4{ }^{\circ} \mathrm{C}$. The precipitate from the ethanol dispersion was collected by centrifugation at $12,000 \times \mathrm{g}$ for $15 \mathrm{~min}$, re-dissolved in distilled water and deproteinised with one fifth volume of Sevag reagent $\left(\mathrm{CHCl}_{3}-\mathrm{BuOH}, 5: 1, v / v\right)$ seven times [23]. The deproteinised solution was then dialysed against distilled water, concentrated and lyophilised to yield deproteinised EPSs.

\section{Critical Micelle Concentration}

The critical micelle concentration is defined as the minimal surfactant concentration needed to form micelles [24]. The critical micelle concentration was determined by measuring surface tension. The test sample was divided into various concentrations: 50, 40, 30, 20, 10, 5, 4, 3, 2, 1, $0.5,0.1,0.05$ and $0 \mathrm{~g} / \mathrm{L}$. Surface tension was measured and graphed against the biosurfactant concentration to observe the changes in surface tension due to surfactant addition. 
Emulsification Index

The purified biosurfactant was obtained and $2 \mathrm{~mL}$ of the test sample (with various biosurfactant concentrations) was combined with $3 \mathrm{~mL}$ of machine oil in a test tube $(16 \times$ $150 \mathrm{~mm}$ ). The mixture was oscillated by vortex for $2 \mathrm{~min}$ and left at room temperature for $24 \mathrm{~h}$. The ratio of the height of the emulsion layer to the total solution was measured and multiplied by 100 to obtain the emulsification index of the tested sample [3]. The emulsification capacities of oils are indicated by the emulsification index, $E_{24}$.

$$
\text { Emulsification index, } E_{24}(\%)=\frac{\text { The height of the total solution }}{\text { The height of the emulsion layer }} \times 100
$$

Characterisation of TKU029 Biosurfactant

\section{Thermal Stability}

The purified biosurfactant at a concentration of $1 \mathrm{mg} / \mathrm{mL}$ was subjected to autoclaving at $121{ }^{\circ} \mathrm{C}$ for $20 \mathrm{~min}$. Prior to and after autoclaving, surface tension and capacity for emulsification were measured. These measurements were made to investigate the influence of high-pressure sterilisation on the biosurfactant properties.

\section{Saline Stability}

To investigate the salt tolerance of the biosurfactant, the biosurfactant solution $(1 \mathrm{mg} / \mathrm{mL})$ was divided into samples and $\mathrm{NaCl}$ solution was added to $0,1,2,3,4$ and $5 \%$. After mixing, each concentration was left standing for $1 \mathrm{~h}$ before surface tension was measured by measuring the contact angle. The capacity for emulsification was measured by oil oscillation.

\section{pH Stability}

To study $\mathrm{pH}$ stability, the $\mathrm{pH}$ of a TKU029 biosurfactant solution $(1 \mathrm{mg} / \mathrm{mL})$ was adjusted to different $\mathrm{pH}$ values $(3,4,5,6,7,8,9,10$ and 11) with $1 \mathrm{~N} \mathrm{NaOH}$ or $1 \mathrm{~N} \mathrm{HCl}$. After $1 \mathrm{~h}$, the surface tension was measured. Capacity for emulsification was measured by oil oscillation.

Antimicrobial Analysis of the Biosurfactant

\section{In Vitro Antibacterial Activity Tests}

The antibacterial activity of the biosurfactant was assayed as follows: the test samples were dissolved in sterilised water and added to $10 \mathrm{~mL}$ of nutrition broth (NB; Difco Laboratories, Detroit, MI) to give a final concentration of $0-5 \mathrm{mg} / \mathrm{mL}$. To the above solutions, $0.05 \mathrm{~mL}$ $\left(10^{5} \mathrm{CFU} / \mathrm{mL}\right)$ of each tested strain (Escherichia coli BCRC13086 and Staphylococcus aureus BCRC10780) was added and then incubated at $30{ }^{\circ} \mathrm{C}$ for $24 \mathrm{~h}$ with shaking at $150 \mathrm{rpm}$. After incubation, the number of viable cells was estimated by measuring the optical density at $660 \mathrm{~nm}$.

\section{In Vitro Antifungal Activity Tests}

Petri plates were filled with $5 \mathrm{~mL}$ of molten PDA pre-cooled to $45{ }^{\circ} \mathrm{C}$ and divided into two groups (triplicate for each). To each plate in the experimental group (E), 
biosurfactant $(5 \mathrm{~mL})$ was added to give a final concentration of $0-10 \mathrm{mg} / \mathrm{mL}$. To the control plates (C), an equal volume of sterile water instead of the test solution was added. After the plates had been cooled, fungal spores $(20 \mu \mathrm{L})$ were placed onto the agar surface. Both groups were incubated for $72 \mathrm{~h}$ at $25{ }^{\circ} \mathrm{C}$. The diameters of the largest and smallest fungal colonies were recorded and their averages were calculated. The inhibition ratios were calculated with the formula

$$
\text { Inhibition ratio }(\%)=(C-E) / C \times 100
$$

where $C$ is the average diameter of the largest and smallest colonies of the control group and $E$ is the average diameter of the largest and smallest colonies of the experimental group. Generally, if the inhibitory ratio was $>20 \%$, the tested strains (Fusarium oxysporum BCRC32121 and Aspergillus fumigatus BCRC30099) would be considered as inhibited.

\section{Assessment of Skin Hydration with EPSs}

Skin hydration was investigated using a Corneometer ${ }^{\circledR}$ CM 825 (Courage and Khazaka, Germany) that was mounted on a Multi Probe Adapter ${ }^{\circledR}$ MPA 5 (Courage and Khazaka). Analysis of the moisture retention capacity of the skin is a simple procedure based on the dielectric constant of the water; it is measured in the superficial layers of the stratum corneum as deep as $10-20 \mu \mathrm{m}$ to ensure that the measurement is not influenced by capillary blood vessels. Capacitance changes depending almost solely upon the water content in the stratum corneum are detected and evaluated. The mean value of five single measurements at five different locations within the test and control areas was calculated. The obtained data were statistically analysed using the Wilcoxon test $(p=0.05)$.

The products studied were propylene glycol/butylene glycol/water $(1.5: 1.5: 1, v / v), 5 \%$ $(w / v)$ purified TKU029 EPSs dissolved in propylene glycol/butylene glycol/water $(1.5: 1.5: 1$, $v / v)$ and hyaluronic acid. Fifteen female volunteers of Asian descent (21 \pm 1 years) with healthy skin in the test and control areas participated in this study. Products were applied to $2-\mathrm{cm}^{2}$ test sites located on the volar forearm, with $0.1 \mathrm{~mL}$ for each application. An adjacent untreated skin area served as a control. Skin hydration was evaluated every $30 \mathrm{~min}$ for $180 \mathrm{~min}$. Before the measurements, the volunteers were acclimated for $30 \mathrm{~min}$ in an environment-controlled room at $20 \pm 1{ }^{\circ} \mathrm{C}$ and $40-50 \%$ relative humidity.

\section{Results and Discussion}

\section{Identification of Strain TKU029}

To characterise strain TKU029, 16S rDNA sequencing and phylogenetic analyses were utilised. According to the analysis of the 16S rDNA gene sequence, TKU029 was most closely aligned to Paenibacillus spp. To further characterise strain TKU029, standard morphological, physiological and biochemical plate analysis showed that strain TKU029 was a Gram-positive and endospore-forming bacillus that grows in both aerobic and anaerobic environments. According to the API 50 CHB identification, TKU029 was most closely related to $P$. macerans, with $99.9 \%$ similarity. The phylogenetic identification and API 50 CHB analysis indicated that strain TKU029 belongs to the species P. macerans. 
Culture Conditions for Biosurfactant and EPS Production

In our preliminary experiments, SPP and SHP were each used as the sole carbon/nitrogen source to investigate the production of biosurfactant and EPSs from P. macerans TKU029. The surface tensions of the media after culturing at $37{ }^{\circ} \mathrm{C}$ for 3 days were 39.51 and $45.89 \mathrm{mN} / \mathrm{m}$ for media containing $1 \%(w / v)$ SPP and $1 \%$ $(w / v)$ SHP, respectively. The total sugar contents of the media after culturing at $37{ }^{\circ} \mathrm{C}$ for 3 days were 952 and $535 \mu \mathrm{g} / \mathrm{mL}$ for media containing $1 \%(w / v)$ SPP and $1 \%$ $(w / v)$ SHP, respectively. These results indicated that SPP was a better substrate for the production of biosurfactant and EPSs by strain TKU029. The effects of various factors, such as concentrations of the carbon source, medium aeration, culture temperature and culture $\mathrm{pH}$, were consecutively evaluated, in single-factor experiments, to establish the best conditions for the production of biosurfactant and EPSs from $P$. macerans TKU029.

\section{Effect of SPP Concentration}

Studies of biosurfactants and EPSs have indicated that the medium composition plays a critical role in biosurfactant and EPS production [25-27]. To select the optimal SPP concentration for biosurfactant and EPS production, 0.5-2 \% $(w / v)$ SPP was added to the basal medium. Bacteria were cultured with medium containing 0.5-2\% $(w / v)$ SPP at $37{ }^{\circ} \mathrm{C}$ for 7 days. As shown in Fig. 1a, the surface tension of the medium containing $1 \%(w / v)$ SPP was $36.35 \mathrm{mN} / \mathrm{m}$ at day 4 ; the surface tension of the medium containing $2 \%(w / v)$ SPP was $36.99 \mathrm{mN} / \mathrm{m}$ at day 3 . The surface tension of the medium containing $1 \%(w / v)$ SPP at day 4 was only slightly lower than that of $2 \%(w / v)$ SPP at day 3 (Fig. 1a). The medium containing $2 \%(w / v)$ SPP at day 3 exhibited a low surface tension $(36.99 \mathrm{mN} / \mathrm{m})$, and the surface tension was consistently more stable than that of the medium containing $1 \%(w / v)$ SPP (Fig. 1a). Because SPP is a very cheap biomaterial and longer culture times consume more energy, $2 \%(w / v)$ SPP was most suitable for the production of biosurfactant from $P$. macerans TKU029. Moreover, increased SPP concentration in the medium led to increased total sugar content. The highest amount of total sugar $(3,235 \mu \mathrm{g} / \mathrm{mL})$ was obtained at $2 \%(w / v)$ SPP (Fig. 1a). The bacteria grew rapidly during the first 4 days, and we also found that biosurfactant and total sugar production was closely related to cell growth (Fig. 1a). The surface tension of the culture medium reached its maximum level (on the third day) after cell growth had reached the exponential phase. The total sugar yield reached its maximum level (on the fifth day) after cell growth had reached the stationary phase. Interestingly, strain TKU029 secreted a biosurfactant during the exponential phase, and the total sugar markedly increased afterwards (Fig. 1a). These results indicate that the production of biosurfactant and EPSs is cell growth-dependent and that P. macerans TKU029 is a promising source of biosurfactant and EPSs. After fermentation, the relative weight of the recovered SPP decreased with increased cultivation time (data not shown). The relative weight of the recovered squid pen was $62 \%$ on the third day. The relative weight of the recovered squid pen decreased to $3 \%$ on the fifth day. The percentage of the relative weight of the recovered squid pen reached its minimum value $(0.2 \%)$ on the seventh day. During the 7 days of cultivation, the SPP concentration decreased gradually in the medium, indicating that most of the SPP in the medium had been utilised for biosurfactant and EPS production. 
(a)

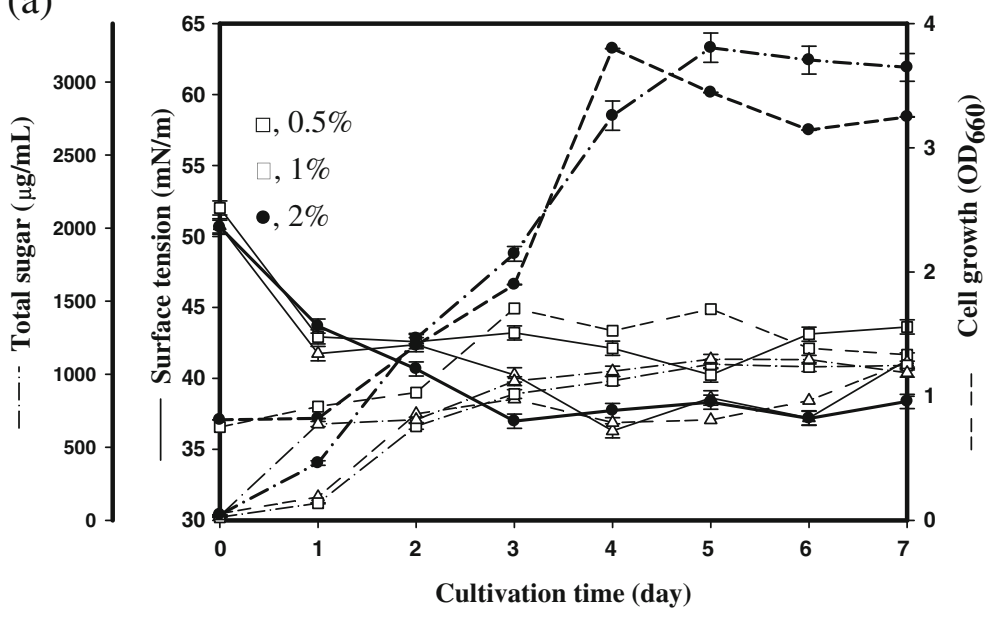

(b)

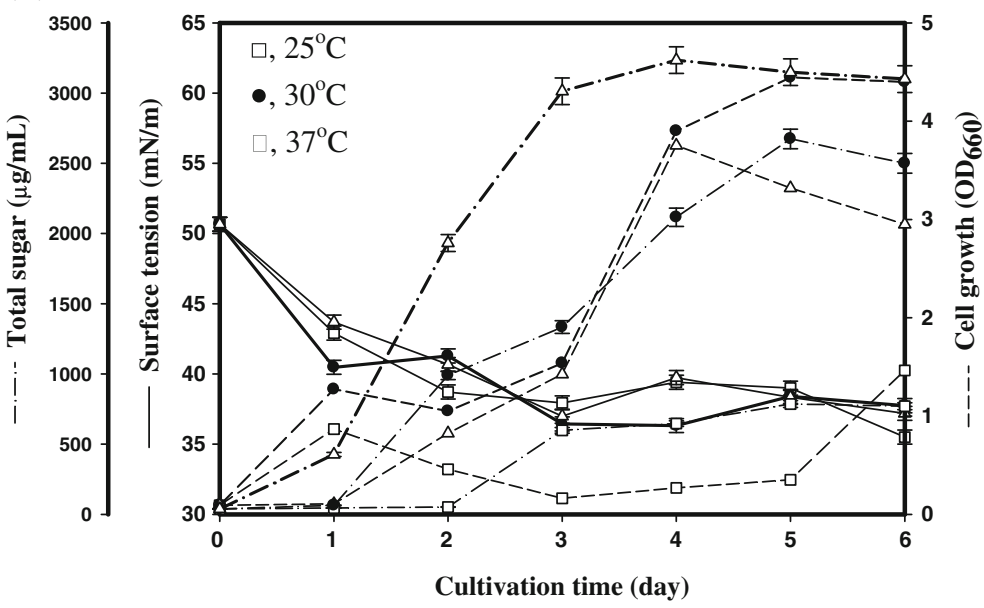

Fig. 1 Effects of SPP suspension (a) and culture temperature (b) on cell growth (dash line), biosurfactant culture medium surface tension (solid line) and EPSs (dash-dot line) production by P. macerans TKU029. All data points are the means $\pm \mathrm{SD}$ of three different experiments performed on different days (each experiment was conducted in triplicate)

\section{Effect of Culture Volume and Temperature}

The importance of oxygenation for biosurfactant and EPS biosynthesis has been well documented $[25,27]$. In this study, with the shaking velocity of a rotary shaker (model LM-570R; Yih Der, Taipei, Taiwan) set to $150 \mathrm{rpm}$, the effect of different volumes of the culture medium in 250-mL Erlenmeyer flasks on the production of biosurfactant and EPSs by P. macerans TKU029 was investigated. Strain TKU029 was inoculated in the above-described medium containing $2 \%(\mathrm{w} / \mathrm{v}) \mathrm{SPP}$. The surface tension values corresponding to volumes of 50, 100 and $200 \mathrm{~mL}$ of the medium were $42.55,36.99$ and $43.51 \mathrm{mN} / \mathrm{m}$, respectively. The total sugar content corresponding to volumes of 50, 100 and $200 \mathrm{~mL}$ of the medium were 2,707,3,235 and $1,688 \mu \mathrm{g} / \mathrm{mL}$, respectively. Therefore, $100 \mathrm{~mL}$ of the medium was more suitable for 
biosurfactant and EPS production than 50 or $200 \mathrm{~mL}$. These results indicated that $P$. macerans TKU029 is a microaerophile that requires oxygen to survive, but requires environments containing lower levels of oxygen than are present in the atmosphere $(\sim 20 \%)$. Under anaerobic conditions, $P$. macerans TKU029 cannot produce biosurfactant (data not shown).

Incubation temperature is another critical factor for biosurfactant and EPS biosynthesis $[25,27]$. Bacteria were cultured in conical flasks under the conditions described above $(2 \%$ $(w / v)$ SPP, $100 \mathrm{~mL}, 150 \mathrm{rpm})$ for 6 days at various temperatures $\left(25,30\right.$ and $\left.37^{\circ} \mathrm{C}\right)$. As shown in Fig. $1 \mathrm{~b}$, the third day of culture at $30^{\circ} \mathrm{C}$ resulted in the optimal and most stable surface tension value $(36.43 \mathrm{mN} / \mathrm{m})$. However, the optimal temperature for EPS production was $37{ }^{\circ} \mathrm{C}$, at which total sugar reached $3,235 \mu \mathrm{g} / \mathrm{mL}$ (Fig. 1b).

\section{Effect of Initial $\mathrm{pH}$}

The initial culture $\mathrm{pH}$ is also an important factor that may affect the cell membrane, cell morphology and structure, the uptake of various nutrients and the biosynthesis of biosurfactant and EPSs [25,27,28]. As shown in Fig. 2, the optimal $\mathrm{pH}$ value for EPS production was 7.21 before sterilisation (i.e. unadjusted $\mathrm{pH}$ ), which corresponded to a total sugar content of $3,235 \mu \mathrm{g} / \mathrm{mL}$ after cultivation. This result was similar to data from Paenibacillus polymyxa strains isolated from soil [29,30], but unlike the slightly alkaline $(\mathrm{pH} 8)$ conditions for EPS production by P. polymyxa EJS-3 [25]. The optimal surface tension activity was reached after 3 days of cultivation at $\mathrm{pH}$ values of 7.21-10 (Fig. 2). After culturing for several more days, the $\mathrm{pH}$ of the culture medium became more alkaline (data not shown). These results indicated that neutral to alkaline $\mathrm{pH}$ conditions were the most suitable for biosurfactant production by strain TKU029. As neutral $\mathrm{pH}$ was more suitable for both biosurfactant and EPS production by TKU029, subsequent experiments were conducted under conditions with a $\mathrm{pH}$ value of 7.21 (unadjusted $\mathrm{pH}$ ).

\section{Time Course of Biosurfactant and EPS Production}

Using SPP as the sole $\mathrm{C} / \mathrm{N}$ source, the relationship between biosurfactant and EPS production was investigated. As shown in Fig. 3, the minimal surface tension $(36.43 \pm 0.42 \mathrm{mN} / \mathrm{m})$

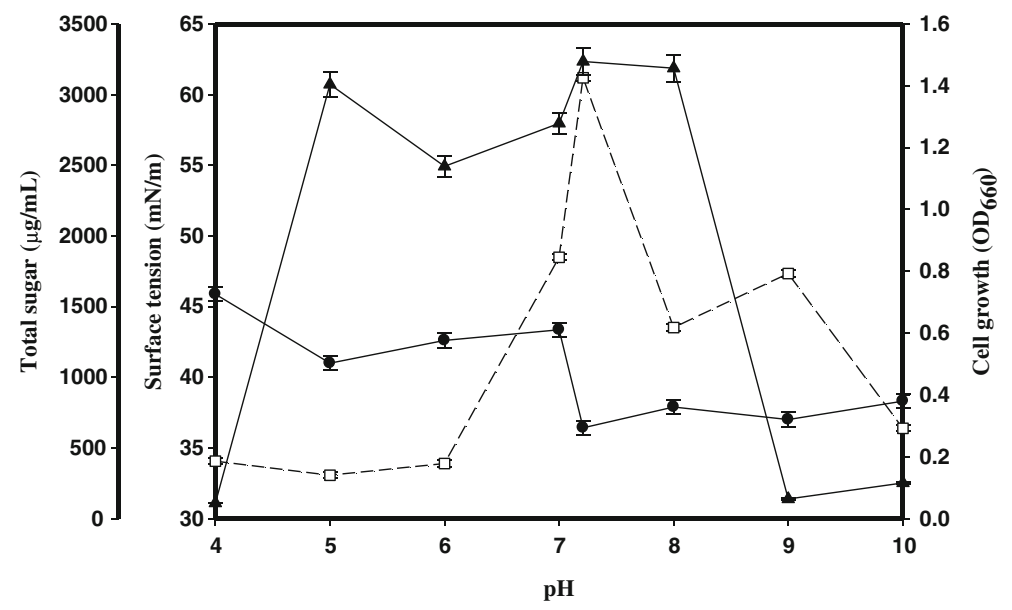

Fig. 2 Effects of initial pH on cell growth (square), biosurfactant culture medium surface tension (circle) and EPS (triangle) production by $P$. macerans TKU029. All data points are the means $\pm \mathrm{SD}$ of three different experiments performed on different days (each experiment was conducted in triplicate) 


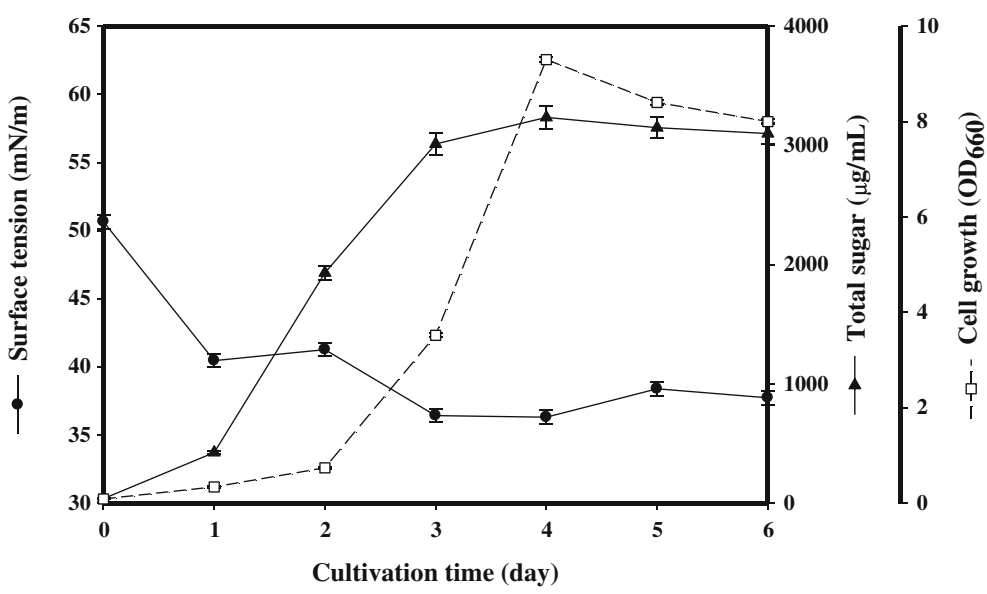

Fig. 3 Time courses of cell growth (square), biosurfactant culture medium surface tension (circle) and EPS (triangle) production in a culture of $P$. macerans TKU029 on squid pen-containing media. All data points are the means $\pm \mathrm{SD}$ of three different experiments performed on different days (each experiment was conducted in triplicate)

of culture medium containing $2 \%(w / v)$ SPP was reached after 3 days of culture at $30{ }^{\circ} \mathrm{C}$. However, the corresponding initial value prior to optimisation (i.e. in medium containing $1 \%(w / v)$ SPP at $37{ }^{\circ} \mathrm{C}$ for 3 days) was $39.51 \pm 0.50 \mathrm{mN} / \mathrm{m}$. Therefore, there was a 3.08 $\mathrm{mN} / \mathrm{m}$ decrease after the optimal culture conditions were established. However, the culture medium alone had an initial surface tension of $50.66 \pm 0.30 \mathrm{mN} / \mathrm{m}$, so that the surface tension decreased by $14.23 \mathrm{mN} / \mathrm{m}$ after culture under optimal conditions. Although this surface tension was higher than that of some biosurfactants, such as the biosurfactant produced by Bacillus subtilis 20B $(29.5 \mathrm{mN} / \mathrm{m})$ [19], it was lower than the surface tension of the biosurfactant produced by Bacillus licheniformis MS3 $(38 \mathrm{mN} / \mathrm{m})$ [2]. Compared with water, the surface tension of TKU029 culture medium after 3 days of cultivation was $36.43 \mathrm{mN} / \mathrm{m}$, a $35.87-\mathrm{mN} / \mathrm{m}$ decrease from the surface tension of water $(72.30 \mathrm{mN} / \mathrm{m})$. This result indicated that the biosurfactant produced by strain TKU029 was effective for reducing surface tension. The surface tension of the culture medium stably remained within the minimum range as cultivation time increased (Fig. 3). This result might be due to saturation of the biosurfactant in the solution or a nearly constant yield of the biosurfactant in the solution. In other words, either the critical micelle concentration or the maximum yield had been reached, and therefore surface tension ceased to decrease. However, the former explanation seems unlikely as the minimal surface tension of the culture medium $(36.43 \pm$ $0.42 \mathrm{mN} / \mathrm{m}$ after 3 days) was $>35.34 \pm 0.22 \mathrm{mN} / \mathrm{m}$, the value measured (in experiments described below) at the critical micelle concentration of $2.76 \mathrm{~g} / \mathrm{L}$. Moreover, the yield of biosurfactant was $1.78 \mathrm{~g} / \mathrm{L}$ in the isolation procedures described below, implying that the critical micelle concentration was not reached in the culture medium. Therefore, the inference is that the reduction of surface tension is related to the maximum yield of the biosurfactant rather than saturation of the biosurfactant in the culture medium. This result differed from the production of a biosurfactant from Pseudomonas aeruginosa S6 [9]. With $P$. aeruginosa S6, the critical micelle concentration was reached in the culture medium; minimal surface tension was reached after 1 day of culture, after which a slow reduction of surface tension was maintained [9]. As shown in Fig. 3, the maximum total sugar content $(3,235 \pm 95 \mu \mathrm{g} / \mathrm{mL})$ was observed in $100 \mathrm{~mL}$ of medium after 4 days, after which it decreased 
gradually. The bacterium grew rapidly during the first 4 days of culture, and we also found that total sugar content was closely related to cell growth. The EPS yield $(3.46 \mathrm{~g} / \mathrm{L})$ reached its maximum level after 4 days and after cell growth reached the stationary phase (Fig. 3). This result indicates that the production of EPSs is cell growth-dependent and that $P$. macerans TKU029 is a promising EPS producer. Among Paenibacillus spp., $P$. polymyxa EJS-3 produced EPSs when growing on $16 \%$ sucrose and $1 \%$ yeast extract as the carbon and nitrogen source [25]. Compared to $P$. polymyxa EJS-3, EPS production by $P$. macerans TKU029 used a cheaper medium. Moreover, in most previous reports, the effect of sugars on the production of biosurfactant was investigated, and it was found that the biosurfactant was quickly produced by adding glucose to the medium [5,7-9,19,31]. However, in this study, $P$. macerans TKU029 adjusted to the culture conditions and could use fish waste SPP as a $\mathrm{C} / \mathrm{N}$ source to produce biosurfactants and EPSs.

\section{Monosaccharide Composition of EPSs}

To determine the monosaccharide composition and estimate the glycosidic bond characteristics of the purified EPSs, the purified EPSs were hydrolysed with $0.5 \mathrm{U} / \mathrm{mL}$ cellulase, pectinase or $\alpha$-amylase at $45{ }^{\circ} \mathrm{C}$ for $24 \mathrm{~h}$. The hydrolysates obtained were separated with TLC. No hydrolysis was observed for EPSs treated with pectinase and $\alpha$-amylase, whereas EPSs treated with cellulase showed two bands on the TLC plates (data not shown). The difference in hydrolysis of the EPSs by cellulase and $\alpha$-amylase implies that the glycosidic bond is most likely a $\beta-1,4$ glycosidic bond rather than an $\alpha-1,4$ glycosidic bond.

\section{Isolation of Biosurfactant}

The literature reports different methods for biosurfactant purification that are dependent on various properties such as acid precipitation [32], cold acetone extraction and electrocoagulation/flotation [2]. This study primarily used alkaline precipitation to purify the biosurfactant, and it employed other methods for initial purification. In method 1, the supernatant was adjusted to $\mathrm{pH} \mathrm{12,} \mathrm{precipitated} \mathrm{and} \mathrm{centrifuged;} \mathrm{the} \mathrm{supernatant} \mathrm{was}$ extracted with ethyl acetate; the organic layer was concentrated using vacuum drying and extracted with methanol; and insoluble substances were removed and concentrated using vacuum drying. In method 2, the supernatant was adjusted to $\mathrm{pH} \mathrm{12,} \mathrm{precipitated} \mathrm{and}$ centrifuged; the supernatant was extracted with ethyl acetate; the organic layer was concentrated using vacuum drying and extracted with acetone; and insoluble substances were removed and concentrated using vacuum drying. In method 3, the supernatant was adjusted to $\mathrm{pH} 12$, precipitated and centrifuged; the pellet was removed; the supernatant was lyophilised and extracted with methanol; and insoluble substances were removed and concentrated using vacuum drying. The purified biosurfactants obtained using the above methods were measured for their ability to reduce surface tension; the results are shown in Fig. 4. Observation of changes in surface tension with different biosurfactant concentrations showed that the surface tension of solutions of the biosurfactant purified using method 3 was the lowest, and it maintained a low value after concentration (Fig. 4). The purified biosurfactant was obtained and weighed using the above-described methods. The biosurfactant yield in the initial culture broth was then calculated. Method 3 produced not only the maximum yield $(1.78 \mathrm{~g} / \mathrm{L})$ of the biosurfactant from the culture broth, but the resulting biosurfactant also showed the lowest surface tension $(36.43 \mathrm{mN} / \mathrm{m})$. Above a TKU029 biosurfactant concentration of $3 \mathrm{mg} / \mathrm{mL}$, no further decrease in surface tension 


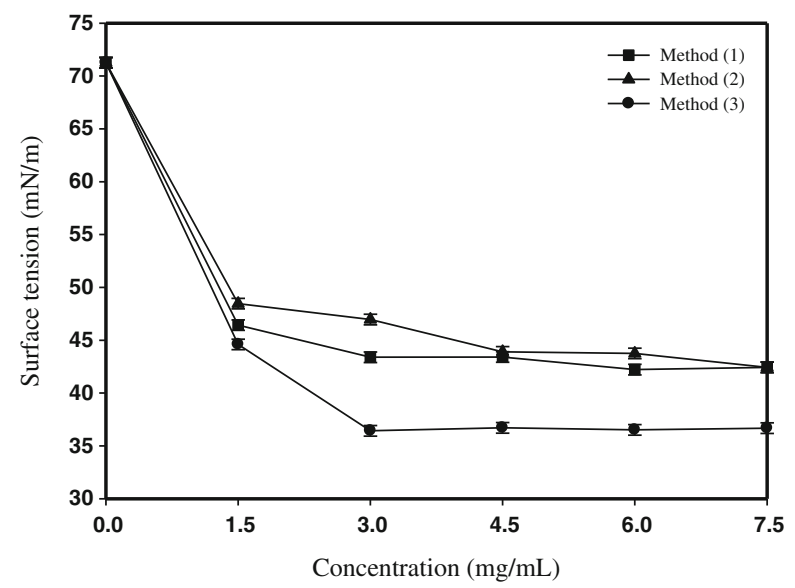

Fig. 4 Surface tension of biosurfactant from P.macerans TKU029 isolated using various procedures. All data points are the means \pm SD of three different experiments performed on different days (each experiment was conducted in triplicate)

was observed (Fig. 4). This outcome indicated that the biosurfactant had reached the critical micelle concentration; therefore, method 3 can be used to produce pure biosurfactants.

Critical Micelle Concentration of the Biosurfactant

The critical micelle concentration (CMC) is the concentration of the biosurfactant at which micelles first begin to form in a solution. $\mathrm{CMC}$ is a critical indicator that can be used for indirect quantification of biosurfactant content by diluting the test sample to various concentrations and measuring the surface tensions of the solutions. When the level of dilution corresponding to the $\mathrm{CMC}$ of the tested sample is determined, the concentration of the biosurfactant in the sample can be indirectly estimated by calculating back from a known CMC value (in this case the value for the strain TKU029 biosurfactant). The CMC was determined by measuring the changes in biosurfactant concentration and surface tension. The surface tension of water gradually decreased from 72.30 to $35.34 \pm$ $0.22 \mathrm{mN} / \mathrm{m}$ with increasing TKU029 biosurfactant concentration; it remained constant above a biosurfactant concentration of $2.76 \mathrm{~g} / \mathrm{L}$ (data not shown). This result was consistent with the other results of this study. How does the CMC of TKU029 biosurfactant compare with other surfactants? Purified surfactin, a biosurfactant produced by B. subtilis PTCC 1696, is more efficient as its CMC was reached at $0.1 \mathrm{~g} / \mathrm{L}$ [4]. The $\mathrm{CMC}$ value of the chemical surfactant SDS is $2.1 \mathrm{~g} / \mathrm{L}$, with a minimum surface tension of $35.41 \mathrm{mN} / \mathrm{m}$. Therefore, the TKU029 biosurfactant is an effective surfactant that is comparable to SDS.

\section{Emulsification Capacity of the Biosurfactant}

The emulsification activity of biosurfactants can be clearly understood through investigation of the emulsification index. Once the emulsification index is determined, this information can be used to estimate the appropriate amount of biosurfactant for use by environmental pollution treatment plants. A small amount of biosurfactant can potentially be used to achieve optimal emulsification effects. Emulsification tests were conducted with machine oil; the capacity for emulsification is shown in Fig. 5. The biosurfactant exhibited an 


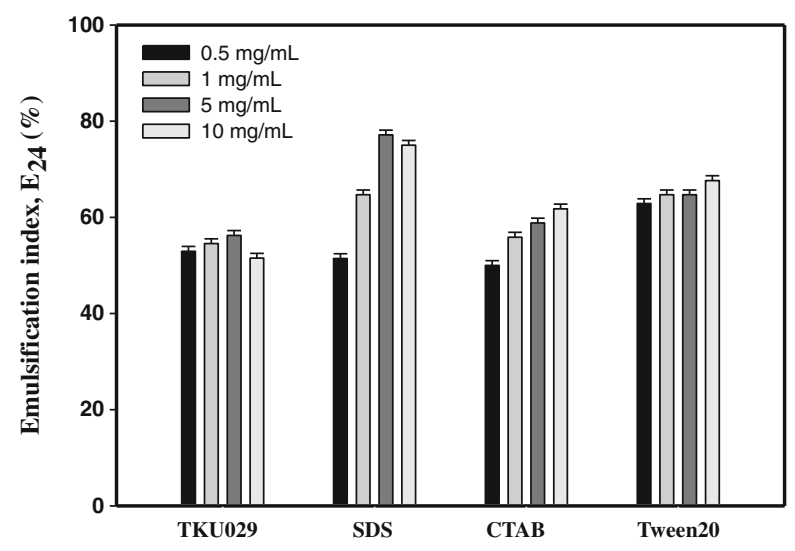

Fig. 5 Emulsion capacity of different surfactants with various concentrations on machine oil. All data points are the means $\pm \mathrm{SD}$ of three different experiments performed on different days (each experiment was conducted in triplicate

emulsification effect on machine oil, and the emulsification index reached 52-56 \% (Fig. 5). Compared to other biosurfactants, the emulsification capacity of the TKU029 biosurfactant is lower than purified surfactin produced by B. subtilis PTCC 1696, which reached an emulsification index of $64.4 \%$ [4]. However, the TKU029-produced biosurfactant had a higher emulsification capacity (56\%) than biosurfactants from B. cereus (48\%) [33] and $B$. licheniformis MS3 (36\%) [2]. The emulsification capacity of various chemical surfactants against machine oil was also tested. The TKU029-produced biosurfactant did not significantly differ from CTAB or Tween 20 in its emulsification capacity (Fig. 5).

\section{Biosurfactant Stability}

\section{Thermal Stability}

Stable biosurfactants are required for the operational and environmental conditions in industry, especially in the petrochemical industry. In microbial-enhanced oil recovery processes, biosurfactants are used as coadjutants, and stable agents are required for the high temperature, pressure and salinity of the oil reservoirs [27]. As shown in Fig. 6a, samples were sterilised at high temperature $\left(121{ }^{\circ} \mathrm{C}, 20 \mathrm{~min}\right)$; the surface tension and capacity for emulsification were measured prior to and after sterilisation. The results showed that the samples did not significantly differ after sterilisation, indicating that the biosurfactant had high thermal stability.

\section{Saline Stability}

This study investigated the feasibility of employing biosurfactants in the treatment of marine oil pollution for potential use in the environmental restoration of sites contaminated by oil. The salinity of ocean water is defined as the gross weight of soluble salts per kilogram, and it often ranges between 3 and $3.7 \%$. This study used saline solutions with concentrations of 0 , 1, 2, 3, 4 and $5 \%$ salt. Each solution was evenly mixed with $1 \mathrm{mg} / \mathrm{mL}$ of sample solution. Surface tension and capacity for emulsification were then measured. As salinity increased, the capacity for emulsification of machine oil did not differ significantly between 48 and 

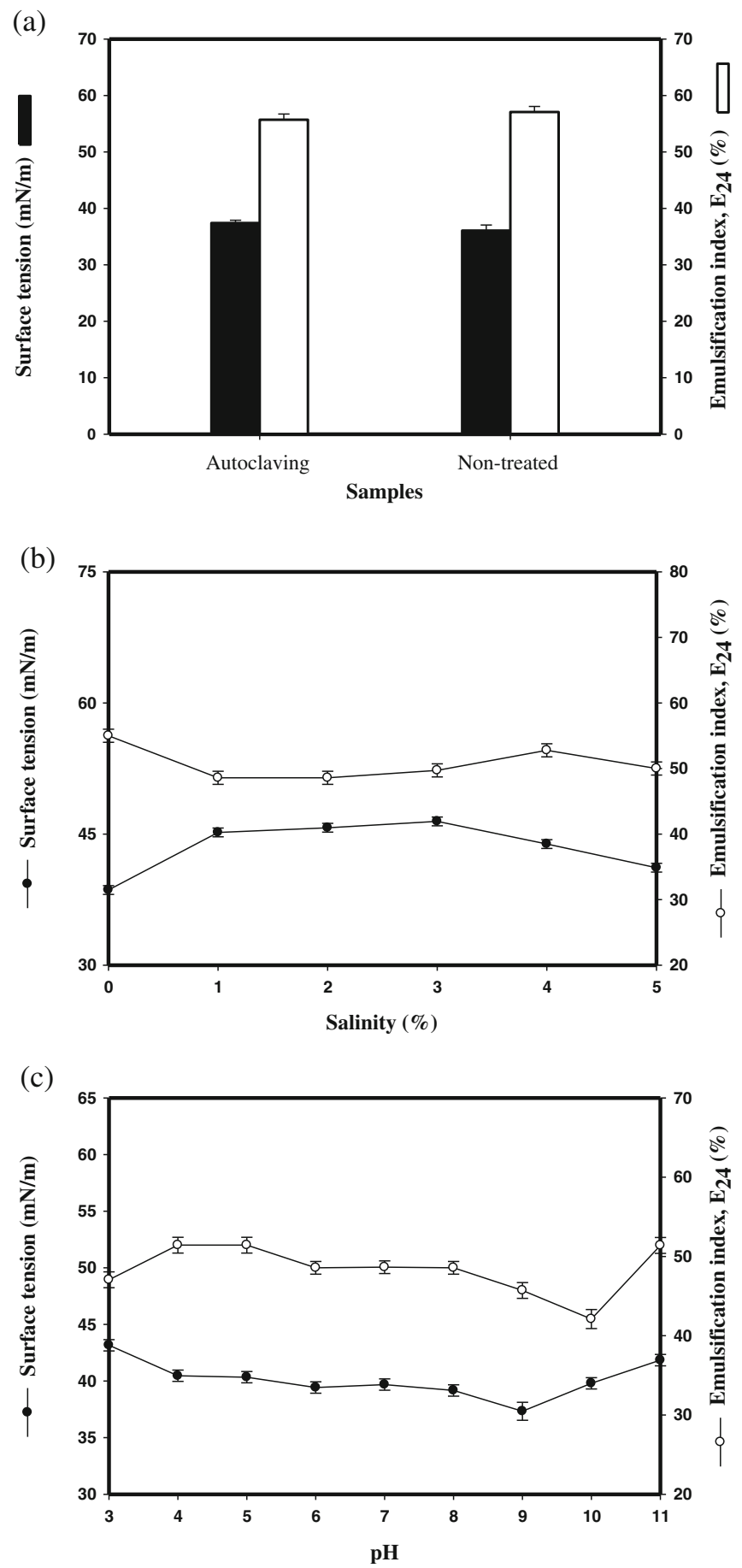

Fig. 6 Effects of heat (a), salinity (b) and $\mathrm{pH}$ (c) on the stability of the surface tension and emulsification capacity of the biosurfactant from $P$. macerans TKU029. All data points are the means \pm SD of three different experiments performed on different days (each experiment was conducted in triplicate) 
$55 \%$, and the surface tension increased from 38.60 to $46.45 \mathrm{mN} / \mathrm{m}$ (Fig. 6b). The reason for these results is presumably the structure of the biosurfactant molecule interacting with physicochemical factors such as salinity to affect micelle formation and shape, therefore affecting the solubility of the biosurfactant and decreasing its activity. The saline stability of the TKU029 biosurfactant is comparable to various types of synthetic surfactants in that there are no differences among the SDS, CTAB, Tween20 and TKU029 biosurfactants (data not shown).

\section{pH Stability}

Studies on the surface tension and emulsification capacity of the TKU029 biosurfactant showed that it was stable over a wide $\mathrm{pH}$ range. An increase in the surface tension was observed at $\mathrm{pH}$ extremes of 3 and 11, suggesting some alteration in the surfactant structure at these $\mathrm{pH}$ values. The emulsification capacity of the TKU029 biosurfactant for machine oil was not significantly different at different $\mathrm{pH}$ values (Fig. 6c). These results showed that the TKU029 biosurfactant is stable in this $\mathrm{pH}$ range for related applications in the future.

\section{Antimicrobial Effects of the TKU029 Biosurfactant}

Today, a number of biosurfactants/bioemulsifiers with antimicrobial activities are available and are used. Hence, these types of surface-active agents have applications in antimicrobial chemotherapy [12]. In previous investigations of antimicrobial effects, iturin A and surfactin produced by B. subtilis could effectively inhibit the plant pathogenic fungi Rhizoctonia solani [19]. The present study included microbial inhibition tests with E. coli BCRC13086, S. aureus BCRC10780, F. oxysporum BCRC32121 and A. fumigatus BCRC30099. The purified TKU029 biosurfactant was added to NB or PDA at concentrations of $0-10 \mathrm{mg} / \mathrm{mL}$ (water was added in the control group). The biosurfactant displayed significant inhibitory effects on E. coli BCRC13086 and S. aureus BCRC10780 at concentrations of 2 and $1.5 \mathrm{mg} / \mathrm{mL}$, respectively (Fig. 7). The half-maximal inhibitory concentrations $\left(\mathrm{IC}_{50}\right)$ of the

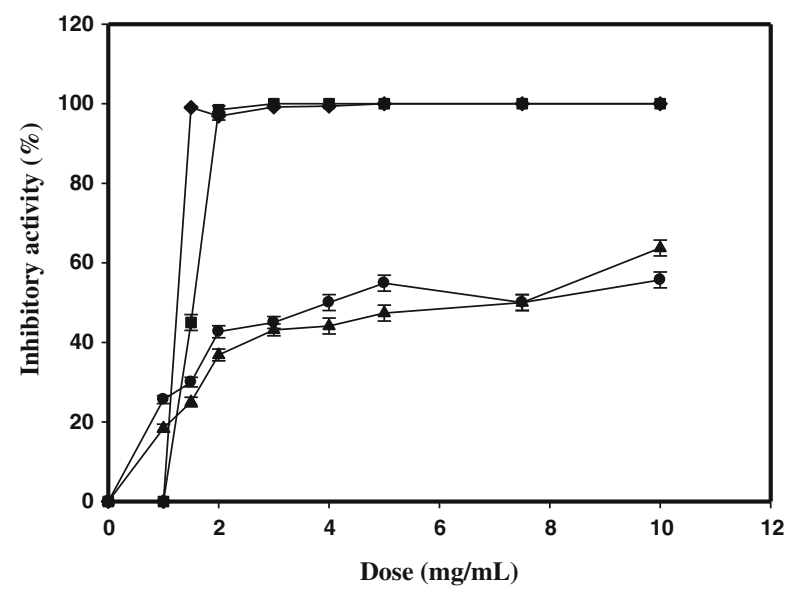

Fig. 7 In vitro growth inhibition of microbes by the biosurfactant from P. macerans TKU029 (circle, F. oxysporum BCRC32121; triangle, A. fumigatus BCRC30099; square, E. coli BCRC13086; diamond, S. aureus $\mathrm{BCRC10780)}$. All data points are the means $\pm \mathrm{SD}$ of three different experiments performed on different days (each experiment was conducted in triplicate) 
biosurfactant against $F$. oxysporum BCRC32121 and A. fumigatus BCRC30099 were 4 and $7.5 \mathrm{mg} / \mathrm{mL}$, respectively (Fig. 7). Additionally, the antimicrobial activity of the biosurfactant was also unaffected after high-temperature treatment $\left(121^{\circ} \mathrm{C}\right)$ and in different $\mathrm{pH}(\mathrm{pH} 4-10)$ conditions. Thus, such biosurfactants can serve as potential new drugs in antimicrobial chemotherapy.

\section{In Vivo Skin Hydration Effect of TKU029 EPSs}

Polysaccharides of bacterial origin are very important in the cosmetic and pharmaceutical industries [12]. One important criterion for the evaluation of cosmetic products is their effect on skin hydration. The aim of this study was to evaluate whether TKU029 EPSs can significantly increase skin hydration after application.

Fifteen female volunteers (average age of 21 years) participated in this study. The same amount of TKU029 EPSs $(5 \%, w / v)$, propylene glycol/butylene glycol/water $(1.5: 1.5: 1, v / v)$ and hyaluronic acid were applied to the skin. Skin hydration was measured in the test and control areas of each volunteer at each time point. At the beginning of the study (30 min), no significant difference in skin hydration was present in the test areas for TKU029 EPSs and hyaluronic acid. The changes in skin capacitance $30 \mathrm{~min}$ after the application of TKU029 EPSs and hyaluronic acid are expressed in Fig. 8 as a percentage increase. After 90 min, a significant difference in the skin hydrating effect of TKU029 EPSs and hyaluronic acid was observed. The TKU029 EPSs increased skin hydration significantly more than hyaluronic acid (Fig. 8). After $180 \mathrm{~min}$, the TKU029 EPSs were still significantly superior to hyaluronic acid with regard to skin hydration (Fig. 8). As shown in Fig. 8, during the application period, TKU029 EPSs increased skin hydration from 37.3 to $44.3 \%$. Application of hyaluronic acid led to a slight increase at the beginning of the period, but then skin hydration decreased from $41.0 \%$ (at $30 \mathrm{~min}$ ) to $37.0 \%$ (at $180 \mathrm{~min}$ ). The untreated controls remained nearly unchanged, i.e. $37.3 \%$ at $0 \mathrm{~min}$ and $36.2 \%$ at $180 \mathrm{~min}$. Inspection of the data with regard to the untreated control revealed an increase in skin hydration after $180 \mathrm{~min}$ of $8.1 \%$ for TKU029 EPSs and of approximately $0.8 \%$ for hyaluronic acid.

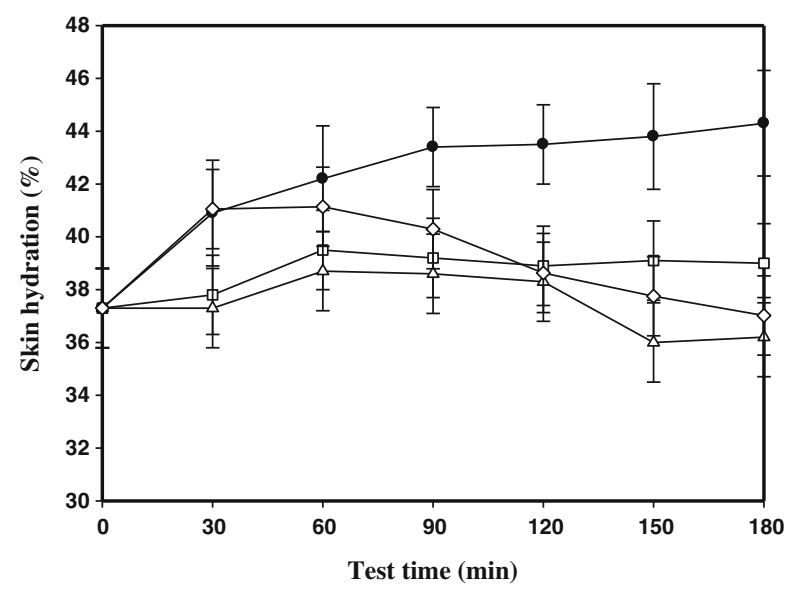

Fig. 8 In vivo skin hydration after application of EPSs from P. macerans TKU029 for 180 min (circle, TKU029 EPS; diamond, hyaluronic acid; square, propylene glycol/butylene glycol/water (1.5:1.5:1, v/v); triangle, control). All data points are the means $\pm \mathrm{SD}$ of three different experiments performed on different days (each experiment was conducted in triplicate) 


\section{Conclusion}

The EPSs produced by strain TKU029 can increase in vivo skin hydration and may be a new source of natural moisturisers with potential value in cosmetics. The biosurfactant produced by P. macerans TKU029 can reduce the surface tension of water to $35.34 \mathrm{mN} / \mathrm{m}$, exhibits an emulsification index $\left(E_{24}\right)$ of $56 \%$ and is stable at high temperature, over a wide $\mathrm{pH}$ range, and at $<5 \%$ salinity. Strain TKU029 thus has potential for the removal of oil from contaminated ocean shores for bioremediation. The TKU029 biosurfactant also has inhibitory effects on microbial growth and thus can potentially be developed as an antimicrobial agent in the future.

Acknowledgments This work was supported in part by a grant of the National Science Council, Taiwan (NSC 101-2621-M-032-005- and NSC 101-2811-M-032-015-).

\section{References}

1. Fiechter, A. (1992). Trends in Biotechnology, 10, 208-217.

2. Biria, D., Maghsoudi, E., Roostaazad, R., Dadafarin, H., Sahebghadam Lotfi, A., \& Amoozegar, M. A. (2009). World Journal of Microbiology and Biotechnology, 26, 871-878.

3. Cooper, D. G., \& Goldenberg, B. G. (1987). Applied and Environmental Microbiology, 53, $224-229$.

4. Ghojavand, H., Vahabzadeh, F., Roayaei, E., \& Shahraki, A. K. (2008). Journal of Colloid and Interface Science, 324, 172-176.

5. Haddad, N. I., Liu, X., Yang, S., \& Mu, B. (2008). Protein and Peptide Letters, 15, 265-269.

6. Haddad, N. I., Wang, J., \& Mu, B. (2008). Journal of Industrial Microbiology \& Biotechnology, 35, 1597-1604.

7. Kim, H. S., Yoon, B. D., Lee, C. H., Suh, H. H., Oh, H. M., Katsuragi, T., \& Tani, Y. (1997). Journal of Fermentation and Bioengineering, 84, 41-46.

8. Pansiripat, S., Pornsunthorntawee, O., Rujiravanit, R., Kitiyanan, B., Somboonthanate, P., \& Chavadej, S. (2010). Biochemical Engineering Journal, 49, 185-191.

9. Yin, H., Qiang, J., Jia, Y., Ye, J., Peng, H., Qin, H., Zhang, N., \& He, B. (2009). Process Biochemistry, 44, 302-308.

10. Gutierrez, J. R., \& Erickson, L. E. (1977). Biotechnology and Bioengineering, 19, 1331-1349.

11. Desai, J. D., \& Banat, I. M. (1997). Microbiology and Molecular Biology Reviews, 61, 47-64.

12. Satpute, S. K., Banat, I. M., Dhakephalkar, P. K., Banpurkar, A. G., \& Chopade, B. A. (2010). Biotechnology Advances, 28, 436-450.

13. Kumar, A. S., Mody, K., \& Jha, B. (2007). Journal of Basic Microbiology, 47, 103-117.

14. Freitas, F., Alves, V. D., Pais, J., Costa, N., Oliveira, C., Mafra, L., Hilliou, L., Oliveir, R., \& Reis, M. A. M. (2009). Bioresource Technology, 100, 859-865.

15. Lin, S. C. (1996). Journal of Chemical Technology and Biotechnology, 66, 109-120.

16. Horowitz, S., Gilbert, J. N., \& Griffin, W. M. (1990). Journal of Industrial Microbiology \& Biotechnology, 6, 243-248.

17. Lin, S. C., Minton, M. A., Sharma, M. M., \& Georgiou, G. (1994). Applied and Environmental Microbiology, 60, 31-38.

18. Youssef, N. H., Duncan, K. E., Nagle, D. P., Savage, K. N., Knapp, R. M., \& Mclnerney, M. J. (2004). Journal of Microbiological Methods, 56, 339-347.

19. Joshi, S., Bharucha, C., \& Desai, A. J. (2008). Bioresource Technology, 99, 4603-4608.

20. Wang, K. Y., Lee, K. T., \& Liao, C. H. (2010). Journal of Marine Science and Technology, 18, 99-105.

21. Wang, S. L., Wu, P. C., \& Liang, T. W. (2009). Carbohydrate Research, 344, 979-984.

22. Dubois, M., Gilles, K. A., Hamilton, J. K., Rebers, P. A., \& Smith, F. (1956). Analytical Chemistry, 28 , $350-356$.

23. Staub, A. M. (1965). Methods in Carbohydrate Chemistry, 5, 5-6.

24. Becher, P. (1965). Emulsions: theory and practice, 2nd ed. (pp. 267-325). New York: Reinhold.

25. Liu, J., Luo, J., Ye, H., Sun, Y., Lu, Z., \& Zeng, X. (2009). Carbohydrate Polymers, 78, $275-281$. 
26. Park, N. H., Choi, H. J., \& Oh, D. K. (2005). Biotechnology Letters, 27, 495-497.

27. Silva, S. N., Farias, C. B., Rufino, R. D., Luna, J. M., \& Sarubbo, L. A. (2010). Colloids and Surfaces B: Biointerfaces, 79, 174-183.

28. Kim, H. O., Lim, J. M., Joo, J. H., Kim, S. W., Hwang, H. J., Choi, J. W., \& Yun, J. W. (2005). Bioresource Technology, 96, 1175-1182.

29. Han, Y. W., \& Clarke, M. A. (1990). Journal of Agricultural and Food Chemistry, 38, 393-396.

30. Lee, I. Y., Seo, W. T., Kim, G. J., Kim, M. K., Ahn, S. G., Kwon, G. S., \& Park, Y. H. (1997). Bioprocess Engineering, 16, 71-75.

31. Arutchelvi, J., Bhaduri, S., Uppara, P. V., \& Doble, M. (2009). Journal of Applied Sciences, 9, 31513155 .

32. Sobrinho, H. B. S., Rufino, R. D., Luna, J. M., Salgueiro, A. A., Campos-Takaki, G. M., Leite, L. F. C., \& Sarubbo, L. A. (2008). Process Biochemistry, 43, 912-917.

33. Velázquez-Aradillas, J. C., Toribio-Jiménez, J., del Carmen Ángeles González-Chávez, M., Bautista, F., Cebrián, M. E., Esparza-García, F. J., \& Rodríguez-Vázquez, R. (2011). World Journal of Microbiology and Biotechnology, 27, 907-913. 